\title{
Predicted Fe I-III fluxes for AGNs with BLRs
}

\author{
T. A. A. Sigut ${ }^{1}$, Anil K. Pradhan ${ }^{2}$ and Sultana N. Nahar ${ }^{2}$ \\ ${ }^{1}$ Department of Physics \& Astronomy, The University of Western Ontario, London, Ontario, \\ Canada email: asigut@uwo.ca \\ ${ }^{2}$ Department of Astronomy, The Ohio State University, Columbus, Ohio, USA
}

\begin{abstract}
We present theoretical Fe I-III emission-line strengths for physical conditions typical of Active Galactic Nuclei with Broad-Line Regions. We can satisfactorily reproduce the empirical UV Fe II-III emission-line template of Vestergaard \& Wilkes (2001) for the prototypical narrowline Seyfert 1 galaxy I Zw 1, although a number of detailed discrepancies remain.
\end{abstract}

\section{Introduction}

The UV spectra of active galactic nuclei (AGN) with broad-line regions (BLRs) exhibit a quasi-continuum of thousands of blended Fe emission lines, dominated by Fe II. However, UV transitions of Fe III are also well established (Laor et al. 1997, Vestergaard \& Wilkes 2001). Laor et al. (1997) identify a strong feature near $\lambda 2418 \AA$ in the spectrum of the prototypical narrow-line Seyfert 1 (NLS1) galaxy I Zw 1 as Fe III multiplet UV47. Vestergaard \& Wilkes (2001) provide a detailed analysis of the UV Fe III emission from I Zw 1 and use their observations to empirically derive Fe II and Fe III flux templates.

\section{Calculations and Discussion}

To predict the Fe line fluxes, the coupled equations radiative transfer and statistical equilibrium were solved following Sigut \& Pradhan (2003). The four lowest ionization stages of Fe, FeI-IV, were explicitly included in the calculations. The final non-LTE atomic model included 944 energy levels and 14,962 radiative transitions. The majority of radiative and collisional atomic data was computed using methods developed under the Iron Project (Hummer et al. 1993). All calculations were performed in models of single BLR clouds computed for a fixed ionization parameter $\left(U_{\text {ion }}\right)$ and total particle density $\left(n_{\mathrm{H}}\right)$.

Among the models considered in Figure 1, the higher density, intermediate ionization parameter model u20h11 does a good job of reproducing the overall level of the Fe IIIII UV emission. In this comparison, the predicted fluxes were normalized to the median template flux between 1800 and $2000 \AA$. The model can account for the relative amount of UV Fe II-Fe III flux from I Zw 1, even with a crude, single-zone model. While inspection of Figure 1 clearly reveals a strong correlation between the model and the observed template, there are several disagreements in detail. In the main Fe III wavelength region, 1800-2000 $\AA$, the relative strengths of individual features are not correctly reproduced. Most notable, however, is the failure of any model to correctly reproduce the strength of the feature near $\lambda 2418 \AA$ identified by Laor et al. (1995) as Fe III multiplet UV47.

We also computed a series of models in which the rather uncertain Fe-H charge exchange reaction rates were omitted. The predicted flux of model u20h96 without chargeexchange reactions is shown in Figure 2. The lack of $\mathrm{Fe}-\mathrm{H}$ charge exchanges reactions shifts the Fe ionization balance in favor of Fe III giving a lower Fe II flux and a higher Fe III flux compared to Figure 1. In fact, normalizing the predicted Fe III fluxes to the 

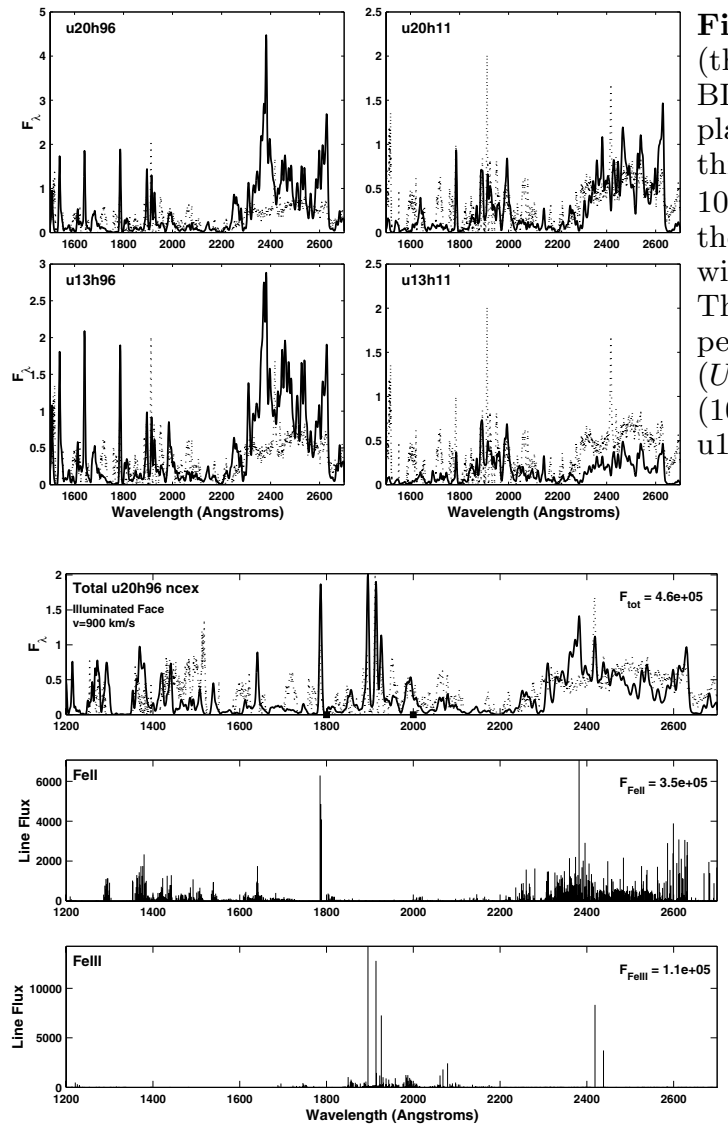

Figure 1. The far-UV Fe II-Fe III flux (thick black line) predicted by four BLR models with the empirical template of Vestergaard \& Wilkes (2001, thin dotted line). $F_{\lambda}$ is in units of $10^{-14} \mathrm{ergs} \mathrm{cm}^{-2} \mathrm{~s}^{-1} \AA^{-1}$. To compare to the template, each model was convolved with a Gaussian of FWHM of $900 \mathrm{~km} \mathrm{~s}^{-1}$. The models are identified in the upper left corner of each panel as: u20h96 $\left(U_{\text {ion }}=10^{-2}, n_{\mathrm{H}}=10^{9.6} \mathrm{~cm}^{-3}\right), \mathrm{u} 20 \mathrm{~h} 11$ $\left(10^{-2}, 10^{11.6}\right)$, u13h96 $\left(10^{-1.3}, 10^{9.6}\right)$, and u13h11 $\left(10^{-1.3}, 10^{11.6}\right)$.

observed template in the interval 1800-2000 $\AA$ results in a much better level of predicted UV Fe II flux as compared to the same model in Figure 1. In addition, the model omitting charge-exchange reactions now predicts strong lines of the Fe III multiplet UV47. However, the predicted flux also contains strong features centered around Fe II $\lambda 2382.04 \AA$ $\left(z^{6} F-a^{6} D\right)$ which have no counterpart (in terms of observed strength) in the empirical template.

Further details of this calculation can be found in Sigut, Pradhan, \& Nahar (2004). We are currently exploring multi-cloud BLR models along the lines of the locally-optimally emitting cloud models of Baldwin et al. (1995), to see if an improved fit to the I Zw 1 UV template can be obtained. We also intend to compare the predictions of such a model to the new optical I Zw 1 template of Véron-Cetty, M.-P., Joly, M., \& Véron (2004, this volume).

\section{References}

Baldwin, J. A., et al. 1995, ApJ, 455, L119

Hummer, D. G., Berrington, K. A., Eissner, W., Pradhan, A. K., Saraph, H. E., \& Tully, J. A. 1993, A\&A, 279, 298

Laor, A., Jannuzi, B. T., Green, R. F., \& Boroson, T. A. 1993, ApJ, 489, 656

Sigut, T. A. A., \& Pradhan, A. K. 2003 ApJS, 145, 15

Sigut, T. A. A., Pradhan, A. K., \& Nahar, S. N. 2004, in press (astro-ph/0401168)

Vestergaard, M., \& Wilkes, B. J. 2001, ApJS, 134, 1 